\title{
A Multi-Agent Collaborative Model for Bayesian Opportunistic Channel Accessibility in Railway Cognitive Radio
}

\author{
Zhijie Yin, Yiming Wang, Cheng $\mathrm{Wu}^{*}$ \\ Urban Railway Transportation Institute, Xiangcheng 215131, Suzhou, China
}

\begin{abstract}
Applying cognitive radio to railway communication systems is a cutting-edge research area. This paper aims to solve the optimization problem of the global channels opportunistic accessibility in railway cognitive radio environments. In particular, we propose an efficient cooperative model for multiple wayside base stations. This model consists of Bayesian inference to calculate the probability of successful transmission on a single station along with team collaboration to maximize network performance within a group of base stations. Instead of only performing the traditional sensing and assigning, the base stations have an ability to learn from the interactions among others and the environment to gain prior knowledge. The base station agents further analyze prior knowledge and perform optimal channel assignment for global network performance. Using our cooperative model of channels opportunistic accessibility, we have shown that the model can also reduce the computational complexity in high-mobility communication environments.
\end{abstract}

Keywords: Channel accessibility, spectrum management, railway cognitive radio, Bayesian Network, multi-agent system

(Submitted on December 27, 2016; Revised on March 2, 2017; Accepted on June 17, 2017)

(C) 2017 Totem Publisher, Inc. All rights reserved.

\section{Introduction}

With the rapid growth of wireless network users, spectrum bands, which were first licensed by the government several decades ago, have become costly resources [1,25]. However, some of the spectrum is sporadically used because of the development of science and technology. The current fixed spectrum allocation policy adopted by government agencies has been unable to handle the spectrum scarcity problem due to the growing demand of spectrum resources [20]. To address this critical issue, the Federal Communication Commission has already given permission to unlicensed users to utilize the licensed spectrum when the licensed users are not in use, known as dynamic spectrum access [7]. This concept is not used to solve the spectrum inefficiency problem, but also drive a new research area: Cognitive Radio.

Applying cognitive radio technique to railways has attracted much interest in the research community and government agencies. To increase train safety and efficiency [2], the Federal Railroad Administration proposed a cognitive radio method to improve the performance of positive train control systems. The French Urbanisme des Radio Communications project was one of the first projects in France and in Europe to raise the problem of the optimization of spectral resources in the Paris region, taking into account the transport field and particularly the urban-guided systems [4].

In this paper, we propose an optimized model through which we can better infer channel accessibility and improve the efficiency of opportunistic channel accessibility within entire railway radio networks that contain multiple base stations. The Bayesian Network is used to model and infer channel accessibility. By doing the inference using multiple prior information

* Corresponding author.

E-mail address: cwu@suda.edu.cn. 
and feedback information, base stations can precisely assign channels to the secondary users (train) and thereby decrease the collision between primary users and secondary users.

Many researchers have greatly contributed to addressing this issue in railway Cognitive Radio (rail-CR), such as spectrum sensing, management technique, licensed user activity prediction, and so on [5,15,21]. However, many methods on modeling channel accessibility or radio resource allocation consider only the performance of a single base station, such as [19,27]. In [19], multi-user opportunistic transmission scheduling is modeled, and at each time slot, the user chooses the best weighted channel. The weight of the channel reflects the long-term system fairness. By only considering the time varying property of the channel, the investigation does not seem to be comprehensive. Location information was considered in [6] and used to perform optimal sensing and power allocation. There is a lack of comprehensive research on multiple base station collaboration for global network performance. Also, the Bayesian Nash equilibrium model is given in [16] to provide the competitively optimal behavior for cognitive radio. [3] provides the Bayesian method in spectrum sensing. [28] shows the Bayesian way in licensed user prediction. Based on these investigations, this paper aims to provide a better communication network for the locomotive and the access points for urban scenarios in a rail-CR environment by establishing a comprehensive multi-agent collaboration model for the Bayesian opportunistic channel accessibility using priori context features.

The rest of the paper is organized as follows. Section 2 presents a formulation of our problem in detail, in which a mathematic model for the channel opportunistic access is given. In section 3, the basic principle of the Bayesian Network is summarized, along with the Bayesian Network based channel accessibility model. In section 4, an example of the rail-CR is given to illustrate the inference of the proposed model. Section 5 concludes the paper.

\section{Related Work}

The growth of wireless communication has created higher demands on future wireless communication network of highspeed rails. Also, multiple sensors deployed along the railway used to monitor different environmental factors are now required for stable data transmission. At high speeds problems like the Doppler shift, lead to fast cell switching and penetration loss, consequently causing frequent spectrum handoffs and further lowering the global performance of network communication $[1,14,17]$. The rail-CR study faces different requirements and obstacles currently in railway communication. However, through our investigation of the literatures above, we could not find one which considers multiple factors in railway communication.

Actually, modeling channel accessibility in railway for quantitative analysis is still a challenge. There are four explanations:

(1) It is difficult to forecast the spectrum holes, as the PU activity is intangible and has certain randomness. However, through long-term observation the recorded history may show the probability of PU occurrence and the corresponding distribution of occupancy duration [22,23].

(2) When the train is communicating using a licensed channel, it may suffer a collision with concurrent unlicensed users like wayside devices. Collisions among the concurrent unlicensed users would lower the quality of service $[13,26]$.

(3) The channel quality may induce error during the train transmission due to the inherent unreliability of the wireless channel. A receiver on the train may receive unnecessary duplicate copies of the same signal in a short period because of multi-path reflections. Instead, the background noise needs a higher reception gain to avoid errors [28].

(4) Frequent spectrum handoffs have a negative impact on link delay and link maintenance. Adjacent agents would coordinate to reduce the unnecessary spectrum handoffs [17].

Considering the uncertainty in railway communication and modeling of the channel accessibility, channel accessibility should be inferred from various available context cues. Lacking sufficient clues would cause high uncertainty in such an inferring process. To address this issue, using multiple clues should be introduced in this inferring process to avoid uncertainty. We pay our attention to the causes of the channel accessibility, and the effects that channel accessibility brings to the communication performance between the trains and base station agents. Standing on the impacts on channel accessibility, we divide these causes into four categories: licensed user activity types, peer device interference, physical factors, and priori information from neighboring agents.

Licensed user activity: When an unlicensed user and a licensed user have a collision while using the same channel in a CR network, the spectrum shared by the unlicensed user would seriously affect the date transmission of the licensed user. This kind of concurrent spectrum occurrence with the licensed user goes against the principle of the protection of licensed 
devices [9]. Hence, for channel accessibility of unlicensed users, the activities of licensed users must be considered. This can be seen as the primary cause of the fluctuations of rail-CR networks.

Peer device interference: Some wayside equipment such as Bluetooth devices, wireless sensor networks, and Ad-Hoc networks along the railway can be another interference to the network. We permit the collision between these peer devices by considering the cooperation and competition in cognitive radio networks, which is also a research area to which a lot of attention has been paid [24].

Physical factor: Some physical factors, such as multi-path reflections, channel gain, thermal noise, propagation loss, transmission power, and reception gain often cause an unpredictable transmission failure. We cannot ignore these physical factors.

Neighbor's information: A base station can get information on its neighboring channel-related information and the working channel of the train through interactions with neighboring base stations. Once taking this information into consideration, the base station would obviously reduce the negative spectrum handoffs.



Fig. 1. Proposed Bayesian Network model using causes and effects.

A large amount of prior knowledge constitutes the causes of spectrum decision for a base station. A value function is used to distinguish the accessibility of each channel. Correspondingly, the effects after choosing a channel can also be used to validate the choice of whether or not to be optimal. We often employ the metric of quality of service as the indicator.

Throughput: After assigning the channel, either locomotive or the access point for urban scenarios will start to transmit data over the channel. By calculating the throughput of the unlicensed users, we can easily judge whether this assignment is good or bad.

Power consumption: The transmitter and receiver of the train or base station will consume more power if the assignment is not good enough [10,11]. For example, if some of the packets are dropped during receiving or the receiver finds out that there are occasional bit flips in the data stream, the transmitter and receiver may consume unnecessary power to retransmit.

Packet Delay: End-to-end delay or one-way delay refers to the time taken for a packet to be transmitted across a network from source to destination. Waiting for the licensed user to leave or a peer device transmission queue would cause packet delay. It is also another significant indicator to measure network performance [18].

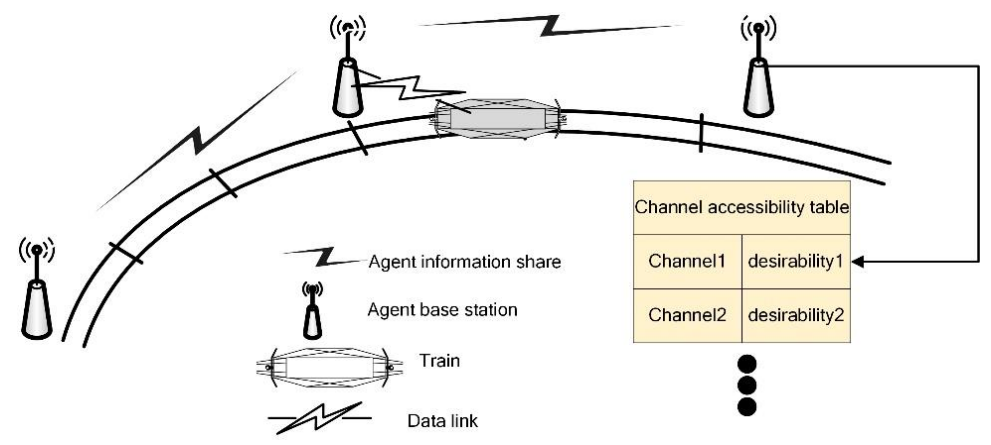

Fig. 2. The overall structure of multi-agent collaborative rail-CR channel accessibility framework. 
With the definition about the causes and effects of channel accessibility, we can explicitly infer the channel accessibility. The Bayesian Network is an effective approach to deal with such uncertainty. It offers reasoning semantics for representing the relation of "cause and effect" via an intuitive graphical representation. The proposed model is shown in Fig. 1. We can see from the figure that the agents can receive information from the environment. Through analyzing and reasoning this information, the agent can assign channels to the trains.

\section{Multi-agent Collaborative Bayesian Opportunistic Channel Accessibility}

The Bayesian Network is a probabilistic graphical model that represents a set of random valuables and their conditional dependencies via a directed acyclic graph. By leveraging the Bayesian Network, we can infer a high-level hypothesis from some observable quantities, latent variables or unknown parameters $[8,12]$. The overall structure of our proposed model along a railway can be seen in Fig. 2 .

\subsection{Bayesian Inference of Channel Opportunistic Accessibility}

Our main purpose of modeling the channel accessibility is to infer the unobservable hypothesis from available context cues. Our modeling for channel accessibility takes 3 steps.

(1) We first need to identify the target hypothesis variable and the corresponding information variable, which have a robust random relation with the target variables.

(2) By building the topology of the directed acyclic graph, we need to divide the information variables node into two categories: "cause" nodes and "effect" nodes according to their casual hierarchical relations with the target node.

(3) Finally, we need to calculate conditional probabilities and infer the results that can help our base station agents make decision.

We evaluate channel accessibility using the probability values over available channels. Now, our target is to infer these probabilities. The influencing factors of channel accessibility and the effects that are caused by the decision made by the base station agent are the information variables. As discussed in Section 2, the influencing factors include

(1) licensed user activity, that is, licensed user arrival probability (LuAP), licensed user departure probability (LuDP), licensed user occupancy time (LuOT), licensed user idle time (LuIT) [6];

(2) peer device interference, that is, bluetooth devices interference (BDI), wireless sensor network interference (WSNI), railway side devices interference (RSDI);

(3) physical factors, that is, channel noise level (CNL), propagation loss (PL), all kinds of fading (FD);

(4) neighboring agent information, that is, the channel that the incoming train was using (CU), locomotive speed (LS), data packet remained in the buffer (DPB).

The effects include throughput (TP), power consumption (PC) and delay (DL) [26].

Combining all of the features, the Bayesian Network model is constructed as shown in Fig. 3. Note that the abbreviations are created just to make the directed acyclic graph and the consequent tables clear and organized.

The next step is to define the states for each node in the direct acyclic graph. For simplicity, in this paper we distinguish the channel accessibility into two different states, that is, successful transmit and failed transmit [8]. We denote successful transmit as the positive state in that it is highly possible for the unlicensed user to successfully transmit on the current channel, and failed transmit as the opposite side of the positive state as the channel status is not qualified for transmission. Once the intermediate node is set, the states of parent nodes and the child nodes can be determined, shown in Table 1 and Table 2 respectively.

In real railway environment, the probabilities of the parent nodes and child nodes can be easily recorded and adjusted by the agents in the long run. Considering that our main purpose is to propose the model in the paper, we here conduct these probabilities of all nodes by subjective estimation.

Now, we can specify the probabilities of each node's states. Let $P=\left\{P_{i} \mid i=1,2, \ldots, 13\right\}$ represents 13 parent nodes of the intermediate node. And let $C=\left\{C_{j} \mid j=1,2,3\right\}$ represents the children nodes. Denote $x$ as the state variable of the intermediate node X. $p_{i}$ and $c_{i}$ are the state variable of the parent nodes $P_{i}$ and child nodes $C_{j}$ respectively. $P\left\{x \mid p_{i}\right\}$ 
and $P\left\{c_{j} \mid x\right\}$ represent the conditional probabilities. The mathematical representation of our proposed model discussed above is shown in Fig. 4.



Fig. 3. Bayesian Network Model for Channel Accessibility.

When the agent receives prior knowledge from the parent and child nodes, denote $e_{p_{i}}$ as the evidence provided by $P_{i}$, and $e_{c_{j}}$ as the evidence provided by $C_{j}$. Then, define the evidence set $e=\left(e_{p_{i}}, e_{c_{j}}\right)$. The hypothesis of the channel accessibility belief is described by the conditional probabilities that $X$ will be in a certain stat after considering the evidence, denoted as $P(x \mid e)$. Considering only the evidence from parent nodes, we can obtain:

$$
P(x \mid e) \propto P\left(x \mid e_{p}\right) \cdot P\left(e_{c} \mid x\right)
$$

According to the Bayesian formula:

$$
\begin{gathered}
P\left(x \mid e_{p}\right)=P\left(x \mid e_{p_{1}}, e_{p_{2}}, \ldots, e_{p_{13}}\right) \\
=\sum_{\text {alli,j,k }} P\left(x \mid p_{1, i}, p_{2, j}, \ldots p_{13, k}\right) \cdot P\left(p_{1, i}, p_{2, j}, \ldots p_{13, k} \mid e_{p_{1}}, e_{p_{2}}, \ldots, e_{p_{13}}\right) \\
=\sum_{\text {alli, }, j, k} P\left(x \mid p_{1, i}, p_{2, j}, \ldots p_{13, k}\right) \cdot P\left(p_{1, i} \mid e_{p_{1}}\right) \ldots P P\left(p_{13, k} \mid e_{p_{13}}\right) \\
=\sum_{\text {allp }} P\left(x \mid p_{m, n}\right) \cdot \prod_{M=1}^{13} P\left(p_{M} \mid e_{p_{M}}\right) \\
P\left(e_{c} \mid x\right)=P\left(e_{c_{1}}, e_{c_{2}}, e_{c_{3}} \mid x\right) \\
=P\left(e_{c_{1}} \mid x\right) P\left(e_{c_{2}} \mid x\right) P\left(e_{c_{3}} \mid x\right) \\
=\prod_{N=1}^{3} P\left(e_{c_{N}} \mid x\right)
\end{gathered}
$$

By substituting equations (2) and (3) into (1): 


$$
P(x \mid e) \propto \prod_{N=1}^{3} P\left(e_{c_{N}} \mid x\right) \cdot\left[\sum_{\text {allp } p_{m, n}} P\left(x \mid p_{m, n}\right) \cdot \prod_{M=1}^{13} P\left(p_{M} \mid e_{p_{M}}\right)\right]
$$

Using equation (4), we can calculate $P(\{x=a\} \mid e)$ and $P(\{x=\bar{a}\} \mid e)$ of the intermediate node state which we denoted above as $\{x=a\}$ and $\{x=\bar{a}\}$. With these inferred results, the agent can easily deduce the state of the current inferring channel by normalizing $P(\{x=a\} \mid e)$ and $P(\{x=\bar{a}\} \mid e)$.

Table 1. State of Parent Nodes

\begin{tabular}{|c|c|c|c|c|c|}
\hline Node property & & Node & & & \\
\hline \multirow{3}{*}{$\begin{array}{l}\text { Licensed user } \\
\text { activity }\end{array}$} & Name & LuAP & LuDP & LuIT & LuOT \\
\hline & \multirow{2}{*}{ State } & High & High & Long & Long \\
\hline & & Low & Low & Short & Short \\
\hline \multirow{3}{*}{$\begin{array}{l}\text { Peer device } \\
\text { interference }\end{array}$} & Name & BDI & RSDI & \multicolumn{2}{|c|}{ WSNI } \\
\hline & \multirow{2}{*}{ State } & Exist & Exist & \multicolumn{2}{|c|}{ Exist } \\
\hline & & Inexist & Inexist & \multicolumn{2}{|c|}{ Inexist } \\
\hline \multirow{3}{*}{ Physical factors } & Name & PL & FD & & \\
\hline & \multirow{2}{*}{ State } & High & High & \multicolumn{2}{|c|}{ High } \\
\hline & & Low & Low & \multicolumn{2}{|c|}{ Low } \\
\hline \multirow{3}{*}{$\begin{array}{l}\text { Neighboring agent } \\
\text { information }\end{array}$} & Name & $\mathrm{LS}$ & DPB & & \\
\hline & \multirow{2}{*}{ State } & Fast & Many & \multicolumn{2}{|c|}{ Current Channel } \\
\hline & & Slow & Less & \multicolumn{2}{|c|}{ Not Current Channel } \\
\hline
\end{tabular}

\subsection{Multi-agent Collaboration Framework}

As we can see in Fig. 2, every agent maintains a channel accessibility table, which acts as the rule for decision-making. Individual actions help the train achieve local optimal performance. Considering global performance, the Bayesian Network on a single agent cannot work perfectly due to the lack of sufficient global information. We provide a multi-agent collaboration framework to solve this problem. After learning when trains pass by, the base station agent receives a value of local desirability to access each channel and the multi-agent system can collect each channel accessibility table. We assume that there are $M$ agents along the railway and $N$ licensed channels can be accessed by the train. Denote $D_{m, n}$ as the $n$th channel's desirability of the $m$ th agent. By normalizing each channel's probability of the agent as the desirability, we have:

$$
G_{n}=\sum_{m=1}^{M} D_{m, n}
$$

as the global desirability of channel $n$. According to the property of the railway, the first agent will rely highly on the global desirability of the expected reward. And the reliability of each agent will decrease when approaching destination, so we have:

$$
C_{m, n}=D_{m, n}+\left(1-\frac{1}{m+1}\right) \cdot G_{n}
$$

Note that $C_{m, n}$ is the desirability of the $n$th channel of the $m$ th agent after collaboration.

Taking channel switch as an example, when the system detects too many switches during cell traveling, a single agent cannot help improve the quality of service in global vision. Through updating the desirability of each channel by performing multi-agent collaboration, the change in desirability will tell the agent to act in a global vision instead of greedily achieving local optimal solution.

Table 2. State of Child Nodes

\begin{tabular}{|c|c|c|c|c|}
\hline \multicolumn{2}{|c|}{ Node } & TP & PC & DL \\
\hline \multirow{3}{*}{ State } & 1 & High & High & High \\
\cline { 2 - 5 } & 2 & Normal & Normal & Normal \\
\cline { 2 - 5 } & 3 & Low & Low & Low \\
\hline
\end{tabular}




\section{Example of Railway Cognitive Radio Communication}

In this section, we extract a simplified single agent channel Bayesian Network model for railway channel accessibility. The simplified channel accessibility model and its mathematical model consist of 13 parent nodes and 3 child nodes, as shown in Fig. 4.



Fig. 4. Mathematical Model of the Channel Accessibility

As discussed above, the paper aims to propose a model, so we assume the conditional probability for each parent and child node as shown in Table 3 and Table 4.

We first consider the case without any evidence by calculating the probability of the state $P(\{x=a\})$ using the data shown in Table 3 as follows:

$$
\begin{aligned}
P(\{x & =a\})=\sum_{\text {allp }_{m, n}} P\left(x \mid p_{m, n}\right) \cdot \prod_{M=1}^{13} P\left(p_{M} \mid e_{p_{M}}\right) \\
& =0.5090
\end{aligned}
$$

and we have:

$$
P(\{x=\bar{a}\})=0.4910
$$

We can see that the results of the good channel accessibility and bad channel accessibility are approximately equal. Without the evidence, the agent cannot determine whether the channel should be assigned to the train.

We assume that the agent starts to record the railway communication information. After sensing every channel and invoking the history, the agent gathers all kinds of information from the environment. See the evidence information from Table 3 in the probability format. After the train passed, we observed that the train gained a fair throughput, cost low power consumption, and had a low delay of transmission. So we set the child nodes $C_{1}, C_{2}, C_{3}$ as $P\left(e_{c_{1}} \mid c_{1,1}\right)=0.5, P\left(e_{c_{1}} \mid c_{1,2}\right)=1.0, P\left(e_{c_{1}} \mid c_{1,3}\right)=0$ and $P\left(e_{c_{2}} \mid c_{2,1}\right)=0, P\left(e_{c_{2}} \mid c_{2,2}\right)=0.5, P\left(e_{c_{2}} \mid c_{2,3}\right)=1.0$ and $P\left(e_{c_{3}} \mid c_{3,1}\right)=0, P\left(e_{c_{3}} \mid c_{3,2}\right)=0.5, P\left(e_{c_{3}} \mid c_{3,3}\right)=1.0$, as shown in Table 4 .

Using the prior probability we can get from the parent nodes, the conditional probability of the $x=a$ and $x=\bar{a}$ can be calculated using equation (2). Using the data conducted above, as:

$$
\begin{aligned}
& P\left(\{x=a\} \mid e_{p}\right)=\sum_{\text {all p }_{m, n}} P\left(x \mid p_{m, n}\right) \cdot \prod_{M=1}^{13} P\left(p_{M} \mid e_{p_{M}}\right) \\
& \quad=1.5353 \\
& P\left(\{x=\bar{a}\} \mid e_{p}\right) \propto 0.2713
\end{aligned}
$$


According to equation (3), the conditional probability of lbegin \{math $\}$ e_clend $\{$ math $\}$ can be calculated as:

$$
\begin{aligned}
& P\left(e_{c} \mid\{x=a\}\right) \propto P\left(e_{c_{1}} \mid x\right) P\left(e_{c_{2}} \mid x\right) P\left(e_{c_{3}} \mid x\right) \\
& \quad=1.85 \\
& P\left(e_{c} \mid\{x=a\}\right) \propto 1.65
\end{aligned}
$$

Integrating equations (9), (10), (11), (12), the conditional probability $P(\{x=a\} \mid e)$ of node $\mathrm{X}$ given all the occurrence of evidences can be calculated as:

$$
\begin{aligned}
P(\{x & =a\} \mid e)=\frac{P\left(\{x=a\} \mid e_{p}\right) P\left(e_{c} \mid\{x=a\}\right)}{P\left(\{x=a\} \mid e_{p}\right) P\left(e_{c} \mid\{x=a\}\right)+P\left(\{x=\bar{a}\} \mid e_{p}\right) P\left(e_{c} \mid\{x=\bar{a}\}\right)} \\
& =0.8639
\end{aligned}
$$

and the conditional probability of $P(\{x=\bar{a}\} \mid e)$ is :

$$
\begin{aligned}
P(\{x & =\bar{a}\} \mid e)=\frac{P\left(\{x=\bar{a}\} \mid e_{p}\right) P\left(e_{c} \mid\{x=\bar{a}\}\right)}{P\left(\{x=a\} \mid e_{p}\right) P\left(e_{c} \mid\{x=a\}\right)+P\left(\{x=\bar{a}\} \mid e_{p}\right) P\left(e_{c} \mid\{x=\bar{a}\}\right)} \\
& =0.1361
\end{aligned}
$$

Conducting the results of the equations (13) and (14), we can see that under the circumstances of the given information,

\begin{tabular}{|c|c|c|c|c|c|c|c|c|c|c|c|}
\hline \multicolumn{2}{|c|}{$P\left(e_{c} \mid x\right)$} & \multirow{2}{*}{$\begin{array}{c}a \\
0.7 \\
\end{array}$} & \multirow{2}{*}{$\begin{array}{c}\bar{a} \\
0.2\end{array}$} & \multicolumn{2}{|c|}{$P\left(e_{c} \mid x\right)$} & \multirow{2}{*}{$\begin{array}{c}a \\
0.3\end{array}$} & \multirow{2}{*}{$\begin{array}{c}\bar{a} \\
0.4\end{array}$} & \multicolumn{2}{|c|}{$P\left(e_{c} \mid x\right)$} & \multirow{2}{*}{$\begin{array}{c}a \\
0.2\end{array}$} & \multirow{2}{*}{$\begin{array}{c}\bar{a} \\
0.1 \\
\end{array}$} \\
\hline \multirow{3}{*}{$C_{1}$} & $C_{1,1}$ & & & \multirow{3}{*}{$C_{2}$} & $C_{2,1}$ & & & \multirow{3}{*}{$C_{3}$} & $C_{3,1}$ & & \\
\hline & $C_{1,2}$ & 0.2 & 0.2 & & $C_{2,2}$ & 0.4 & 0.4 & & $C_{3,2}$ & 0.6 & 0.3 \\
\hline & $C_{1,3}$ & 0.1 & 0.6 & & $C_{2,3}$ & 0.3 & 0,2 & & $C_{3,3}$ & 0.2 & 0.6 \\
\hline
\end{tabular}
the agent can easily tell the channel accessibility. Although the given parts of evidence may be inaccurate, the status of channel accessibility can be clearly inferred in terms of likelihood probabilities. This proves that our proposed model can certainly give a precise inference when the agent has comprehensive information. Note that when the evidence is more precisely given, the more reliable our model will perform.

Table 3. Conditional probabilities for parent nodes

\begin{tabular}{|c|c|c|c|c|c|c|c|c|c|c|c|c|c|}
\hline$P\left(x \mid e_{p}\right)$ & $p_{1,1}$ & $p_{2,1}$ & $p_{3,1}$ & $p_{4,1}$ & $p_{5,1}$ & $p_{6,1}$ & $p_{7,1}$ & $p_{8,1}$ & $p_{9,1}$ & $p_{10,1}$ & $p_{11,1}$ & $p_{12,1}$ & $p_{13,1}$ \\
\hline$a$ & 0.2 & 0.75 & 0.4 & 0.6 & 0.3 & 0.1 & 0.4 & 0.3 & 0.2 & 0.2 & 0.3 & 0.4 & 0.7 \\
\hline $\bar{a}$ & 0.8 & 0.25 & 0.6 & 0.4 & 0.7 & 0.9 & 0.6 & 0.7 & 0.8 & 0.8 & 0.7 & 0.6 & 0.3 \\
\hline$P\left(x \mid e_{p}\right)$ & $p_{1,2}$ & $p_{2,2}$ & $p_{3,2}$ & $p_{4,2}$ & $p_{5,2}$ & $p_{6,2}$ & $p_{7,2}$ & $p_{8,2}$ & $p_{9,2}$ & $p_{10,2}$ & $p_{11,2}$ & $p_{12,2}$ & $p_{13,2}$ \\
\hline$a$ & 0.7 & 0.65 & 0.6 & 0.4 & 0.7 & 0.9 & 0.6 & 0.7 & 0.8 & 0.8 & 0.7 & 0.6 & 0.3 \\
\hline $\bar{a}$ & 0.3 & 0.35 & 0.4 & 0.6 & 0.3 & 0.1 & 0.4 & 0.3 & 0.2 & 0.2 & 0.3 & 0.4 & 0.7 \\
\hline
\end{tabular}

\section{Simulation Results}

In this section, we formulate some simulations in order to verify the effectiveness of our proposed BN scheme. Our overall aim is to best predict the channel accessibility for cognitive users. The simulation is constructed as follows. For simplicity, the CR network contains 1 agent base station and 1 cognitive user. Within the cover range of the agent base station there are 10 channels that are allocated to 10 PUs respectively. All channels are assumed to have the same bandwidth. According to the assumption of the parent nodes above, we model 10 channels using different parameters in order to show the distinction between them. The PU model is subject to the continuous time Markov process. Simulation lasts 2000 seconds. The spectrum-sensing module of our proposed agent base station will notice changes in the environment and update the channel accessibility in real-time, as well as use the QoS to adjust the channel accessibility. Through iteration, the agent base station will predict the channel accessibility more precisely. We separately simulate the BN network with and without the QoS of the cognitive to see if the prior knowledge works well. Also, we compare the result of our proposed scheme with the Reinforcement Learning method in [26] an opportunistic method. 


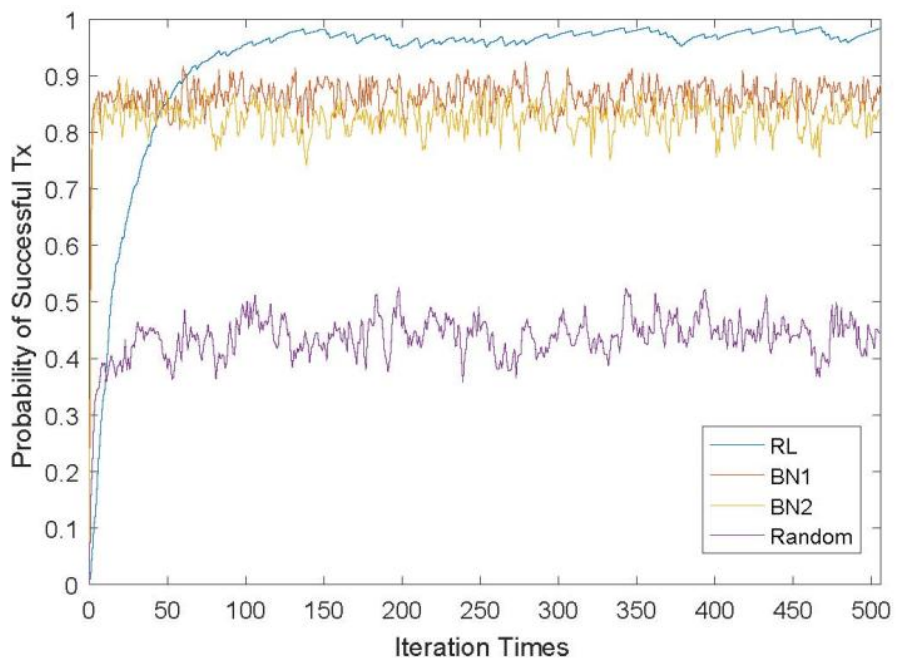

Fig. 5. Probability of Successful Transmission of the 4 different methods in the cognitive network. BN1 represents the proposed method. BN2 is the method without the QoS contextual inference. RL represents the reinforcement learning proposed in [26]. The Random curve represents that the agent base station accessing the channel using opportunistic way.

The probability of successful transmission plot is shown in Fig. 5. The performance curves are averages of over 500 Monte Carlo iterations. It is obvious that the RL method, which conducts its optimal policy during trial-and-error, performs best. This method works well but the learning process lasts long. During the learning process, this method obtains the optimal policy through an exhaustive search of all possible joint actions, which is not effective. We can see from the BN2 curve that our proposed BN network learns faster than the RL method only with the prior knowledge. However, the eventual performance after the initial learning phase is worse than that of the RL method. This gap shows the difference between prediction and the trial-and-error method. We sacrifice some QoS to greatly improve the effectiveness of our system. At the end, the RL method reaches nearly $96 \%$, while the BN method only with prior knowledge reaches $82 \%$. When we add the QoS to help predict the channel accessibility, as shown by curve BN1, the performance of our proposed scheme rises to about $88 \%$, which shows the necessity of the contextual way of updating the channel accessibility. The probability of successful transmission of the opportunistic way of accessing the channel finds averages to $46 \%$.

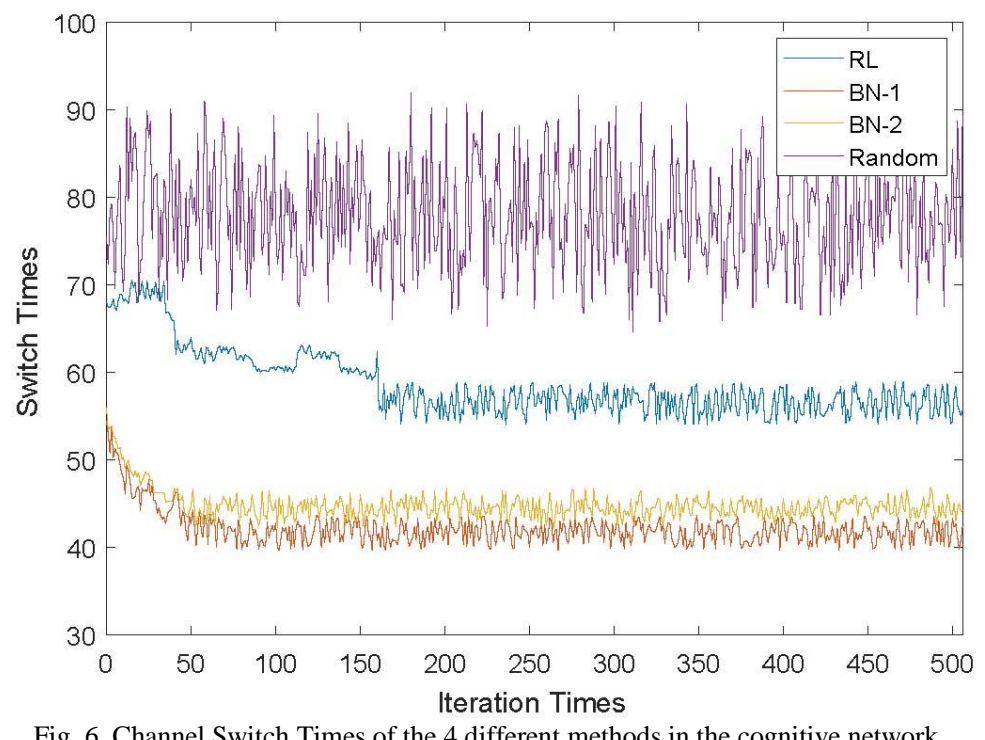

This QoS is different from the indicator in Fig. 5. Channel switch will cause a lot of harm to the communication quality, including switch delay, increased power consumption during reconnection, and even a lost connection. Our proposed method can greatly reduce the channel switch times.

Fig. 6 depicts the channel switch times of different methods. In Fig. 6, the proposed BN1 method shows the best performance among all of the methods. We observe that the RL algorithm chose to trade off the channel switch times with 
the probability of successful transmission. The performance is roughly 55 times after the initial learning phase. Still, the learning phase lasts more than 150 iterations, which shows that the RL method has a long learning phase in order to catch up with the BN method. The BN2 method had a slightly worse performance compared to that of the BN1 method. Moreover, the opportunistic method shows its instability, which shakes between 70 times to 90 times.

The simulation results show that our proposed method has a short learning phase. The BN method can quickly come into use with a good performance. The RL method shows the best probability of a successful transmission, however, it performs a lot of channel switches, which will cost the switch delay and affect the QoS of cognitive user. During the comparison of BN1 and BN2, we can see that the QoS can help infer the channel accessibility. Hence, the BN channel accessibility inference method shows its effectiveness.

\section{Conclusion}

In this paper we propose a Bayesian Network based multi-agent railway communication model for channel accessibility using a fusion of prior and validated information. This alternative for the agent base station can easily infer the channel accessibility for the train in rail-CR environments. Due to its briefness, this approach can be easily deployed in the base station to ensure the communication quality for train control or for civil use. Future work can be directed to further investigation on machine learning paradigm in rail-CR.

\section{References}

1. B. Ai, X. Cheng, T. Kurner, and Z. D. Zhong, "Challenges Toward Wireless Communications for High-Speed Railway," IEEE Trans. Intell. Transp. Syst., vol. 15, no. 5, pp. 2143-2158, 2014.

2. A. Amanna, M. Gadhiok, M. J. Price, J. H. Reed, W. P. Siriwongpairat, and T. K. Himsoon, "Railway Cognitive Radio," IEEE Veh. Technol. Mag., vol. 5, no. 3, pp. 82-89, 2010.

3. M. Balc saran, S. Erküçük, and H. A. Çırpan, "Bayesian compressive sensing for primary user detection," Iet Signal Process., vol. 10, no. 5, pp. 514-523, 2016.

4. M. Berbineau et al., "Cognitive radio for high speed railway through dynamic and opportunistic spectrum reuse," in Transport Research Arena (TRA) 5th Conference: Transport Solutions from Research to Deployment, 2014.

5. B. Canberk, I. F. Akyildiz, and S. Oktug, "Primary User Activity Modeling Using First-Difference Filter Clustering and Correlation in Cognitive Radio Networks," IEEE/ACM Trans. Netw., vol. 19, no. 1, pp. 170-183, 2011.

6. S. Choi, H. Park, and T. Hwang, "Optimal Beamforming and Power Allocation for Sensing-Based Spectrum Sharing in Cognitive Radio Networks," IEEE Trans. Veh. Technol., vol. 63, no. 63, pp. 412-417, 2014.

7. Federal Communications Commission, "Spectrum Policy Task Force Report," Rep. Docket no. 02-135., 2002.

8. N. Friedman, G. Dan, and M. Goldszmidt, "Bayesian Network Classifiers," Mach. Learn., vol. 29, no. 2-3, pp. 131-163, 1997.

9. S. Haykin, "Cognitive radio: brain-empowered wireless communications," IEEE J. Sel. Areas Commun., vol. 23, no. 2, pp. 201220, 2006.

10. A. He, S. Srikanteswara, J. H. Reed, and X. Chen, "Minimizing Energy Consumption Using Cognitive Radio," vol. 22, no. 6, pp. 372-377, 2008.

11. A. He, S. Srikanteswara, K. K. Bae, and T. R. Newman, "System power consumption minimization for multichannel communications using cognitive radio," in IEEE International Conference on Microwaves, Communications, Antennas and Electronics Systems, 2009, pp. 1-5.

12. D. Heckerman, A Tutorial on Learning with Bayesian Networks. MIT Press, 1999.

13. L. Hong-Jiang and Z. Qi, "Tradeoff between utilization and collision in cognitive radio," in International Conference on Wireless Communications \& Signal Processing, 2009, pp. 1-5.

14. M. A. Kalil, H. Al-Mahdi, and A. Mitschele-Thiel, "Spectrum handoff reduction for cognitive radio ad hoc networks," pp. 1036-1040, 2010.

15. I. Kim and D. Kim, "Distributed spectrum sensing and access with secondary channel quality in cognitive radio networks," in The International Conference on Advanced Communication Technology, 2010, pp. 541-546.

16. V. Krishnamurthy, "Decentralized Spectrum Access Amongst Cognitive Radios---An Interacting Multivariate Global GameTheoretic Approach," IEEE Trans. Signal Process., vol. 57, no. 10, pp. 3999-4013, 2009.

17. K. Kumar, A. Prakash, and R. Tripathi, "Spectrum Handoff in Cognitive Radio Networks: A Classification and Comprehensive Survey," J. Netw. Comput. Appl., vol. 35, no. 8, pp. 2501-2511, 2015.

18. S. C. Lin, "End-to-end delay reduction via in-network computation in cognitive radio sensor networks," in Global Communications Conference (GLOBECOM), 2013 IEEE, 2013, pp. 408-413.

19. X. X. Liu, E. K. P. Chong, and N. B. Shroff, "Opportunistic transmission scheduling with resource-sharing constraints in wireless networks," IEEE J. Sel. Areas Commun., vol. 19, no. 10, pp. 2053-2064, 2001.

20. Z. Ma, Z. Q. Zhang, Z. G. Ding, P. Z. Fan, and H. C. Li, "Key techniques for $5 \mathrm{G}$ wireless communications: network architecture, physical layer, and MAC layer perspectives," Sci. China, vol. 58, no. 4, pp. 1-20, 2015.

21. D. Niyato, E. Hossain, and Z. Han, "Dynamics of Multiple-Seller and Multiple-Buyer Spectrum Trading in Cognitive Radio Networks: A Game-Theoretic Modeling Approach," IEEE Trans. Mob. Comput., vol. 8, no. 8, pp. 1009-1022, 2008.

22. Y. Saleem and M. H. Rehmani, "Primary radio user activity models for cognitive radio networks: A survey," J. Netw. Comput. 
Appl., vol. 43, no. 1, pp. 1-16, 2014.

23. K. W. Sung, S. L. Kim, and J. Zander, "Temporal spectrum sharing based on primary user activity prediction," IEEE Trans. Wirel. Commun., vol. 9, no. 12, pp. 3848-3855, 2010.

24. E. Z. Tragos, S. Zeadally, A. G. Fragkiadakis, and V. A. Siris, "Spectrum Assignment in Cognitive Radio Networks: A Comprehensive Survey," IEEE Commun. Surv. Tutorials, vol. 15, no. 3, pp. 1108-1135, 2013.

25. T. Wang, G. Li, J. Ding, and Q. Miao, "5G Spectrum: is china ready?," Commun. Mag. IEEE, vol. 53, no. 7, pp. 58-65, 2015.

26. C. Wu, K. Chowdhury, M. Di Felice, and W. Meleis, "Spectrum management of cognitive radio using multi-agent reinforcement learning," in International Conference on Autonomous Agents and Multiagent Systems, 2010, pp. 1705-1712.

27. R. Xie, F. R. Yu, H. Ji, and Y. Li, "Energy-Efficient Resource Allocation for Heterogeneous Cognitive Radio Networks with Femtocells," IEEE Trans. Wirel. Commun., vol. 11, no. 11, pp. 3910-3920, 2012.

28. X. Xing, T. Jing, Y. Huo, H. Li, and X. Cheng, "Channel quality prediction based on Bayesian inference in cognitive radio networks," in IEEE INFOCOM, 2013, pp. 1465-1473. 